\title{
Uma metodologia de análise da relação entre infraestrutura e incidência de conflitos
}

\author{
Una metodología de \\ análisis de la relación entre infraestructura e incidencia de conflitos
}
An analysis methodology of the relationship between infrastructure and conflict incidence

\author{
José de Ribamar Sousa Pereira ${ }^{1}$ \\ Glória Maria Santiago Pereira ${ }^{2}$ \\ Kelly Tatiane Martins Quirino ${ }^{3}$ \\ Benedito Rodrigues dos Santos ${ }^{4}$
}

\begin{abstract}
Resumo
Diversos estudos procuraram mostrar a ligação entre pobreza e a incidência de conflitos como um ciclo vicioso no qual a pobreza gera conflitos e conflitos geram pobreza. Dessa forma, é imprescindível pensar que, no esforço da análise e prevenção de conflitos, devem ser levadas em ampla consideração as ameaças provenientes dos aspectos estruturais - sociais e econômicos historicamente reproduzidos nos países menos desenvolvidos. Nesse contexto, o presente trabalho busca propor uma metodologia quantitativa para avaliar a relação entre investimento em infraestrutura e possibilidade de incidência de conflitos. Para tanto, considerando-se um período de 2001 a 2016, procurou-se estudar empiricamente a relação entre a incidência de conflitos e investimentos em infraestrutura em setores-chave da economia, tais quais energia elétrica, telecomunicações e transportes, em uma região pré-definida, por meio de estimativas com dados de painel. Com isso, obteve-se evidências empíricas indicando que investimentos, no que se conhece como logística pública e privada, podem contribuir para o crescimento do PIB, redução da pobreza e, por consequência, reduzir a incidência de conflitos. Mais especificamente, os resultados econométricos dessa análise revelaram um significante efeito conjunto de investimentos na referida infraestrutura sobre a ocorrência de conflitos. Finalmente, conclui-se a metodologia e, dessa forma, o objetivo desse artigo - com a demonstração de que as condições estruturais podem representar as raízes dos conflitos em que se envolvem os países que se escolha analisar.
\end{abstract}

Palavras-Chave: Metodologia; Investimento em infraestrutura; Pobreza e conflitos.

\section{Resumen}

Diversos estudios han intentado mostrar el vínculo entre pobreza y la incidencia de conflictos como un ciclo vicioso en el que la pobreza genera conflictos y conflictos generan pobreza. De esta forma, es imprescindible pensar que, en el esfuerzo de análisis y prevención de conflictos, deben ser tomadas en amplia consideración las amenazas provenientes de los aspectos estructurales - sociales y económicos históricamente reproducidos en los

1 Doutorado em economia pela UCB; Ministério da Defesa; Brasília, DF, Brasil; financasribamar@yahoo.com.br. Trabalho apresentado no I Seminário Latino-Americano de Estudos em Cultura - SEMLACult, Foz do Iguaçu/PR, Brasil, 2017.

2 Mestre em psicologia pela UCB; UNIEURO; Brasília, DF, Brasil; gloriappereira5@gmail.com. Trabalho apresentado no I Seminário Latino-Americano de Estudos em Cultura - SEMLACult, Foz do Iguaçu/PR, Brasil, 2017.

3 Doutorado em jornalismo pela UNB; Fundação Banco do Brasil; Brasília, DF, Brasil; itsanacha@yahoo.com.br. Trabalho apresentado no I Seminário Latino-Americano de Estudos em Cultura SEMLACult, Foz do Iguaçu/PR, Brasil, 2017.

$4 \quad$ Doutorado em antropologia pela Universidade da Califórnia Berkeley; Universidade Católica de Brasília; Brasília, DF, Brasil; benesantos989@gmail.com. Trabalho apresentado no I Seminário Latino-Americano de Estudos em Cultura - SEMLACult, Foz do Iguaçu/PR, Brasil, 2017. 
países menos desarrollados. En este contexto, el presente trabajo busca proponer una metodología cuantitativa para evaluar la relación entre inversión en infraestructura y posibilidad de incidencia de conflictos. Para ello, considerando un período de 2001 a 2016, se intentó estudiar empíricamente la relación entre la incidencia de conflictos e inversiones en infraestructura en sectores clave de la economía, tales como energía eléctrica, telecomunicaciones y transportes, en una región pre-Por medio de estimaciones con datos de panel. Con ello, se obtuvieron evidencias empíricas indicando que las inversiones, en lo que se conoce como logística pública y privada, pueden contribuir al crecimiento del PIB, la reducción de la pobreza y, por consiguiente, reducir la incidencia de conflictos. En concreto, los resultados econométricos de este análisis revelaron un significativo efecto conjunto de inversiones en dicha infraestructura sobre la ocurrencia de conflictos. Finalmente, se concluye la metodología -y de esa forma el objetivo de ese artículo- con la demostración de que las condiciones estructurales pueden representar las raíces de los conflictos en que se involucra a los países que se elige analizar.

Palabras clave: Metodología; Inversión en infraestructura; Pobreza y conflictos.

\begin{abstract}
Several studies have tried to show the link between poverty and the incidence of conflicts as a vicious cycle in which poverty generates conflicts and conflicts generate poverty. Thus, it is essential to think that, in the effort to analyze and prevent conflicts, the threats arising from the structural - social and economic aspects historically reproduced in less developed countries should be taken into account. In this context, the present work seeks to propose a quantitative methodology to evaluate the relationship between investment in infrastructure and the possibility of conflict incidence. In order to do so, considering a period from 2001 to 2016, we sought to empirically study the relationship between the incidence of conflicts and investments in infrastructure in key sectors of the economy, such as electricity, telecommunications and transport, in a pre-Defined, using estimates with panel data. Thus, empirical evidence has been obtained indicating that investments, in what is known as public and private logistics, can contribute to GDP growth, reduce poverty and, consequently, reduce the incidence of conflicts. More specifically, the econometric results of this analysis revealed a significant joint effect of investments in such infrastructure on the occurrence of conflicts. Finally, the methodology - and, thus, the objective of this article - is concluded, with the demonstration that structural conditions can represent the roots of the conflicts in which the countries to be analyzed are involved.
\end{abstract}

Keywords: Methodology; Investment in infrastructure; Poverty and conflict.

\title{
1 - Introdução
}

Mostrar a ligação entre pobreza e a incidência de conflitos, como um ciclo vicioso, pode ser uma abordagem viável a fim de obter evidências de que a pobreza gera conflitos e conflitos geram pobreza. Mas, é imprescindível pensar que, no esforço de análise e prevenção de conflitos, devem ser levadas em ampla consideração as ameaças provenientes dos aspectos estruturais sociais e econômicos historicamente reproduzidos nos países menos desenvolvidos.

Tem sido uma preocupação constante, em especial, dos planejadores de políticas públicas, a correlação entre as dotações de infraestrutura e o desempenho da economia, a fim de melhor alocar os recursos públicos. Dado que não se pode prescindir de uma provisão adequada do capital público quando se objetiva uma trajetória de desenvolvimento equilibrada, alguns trabalhos acadêmicos têm sido desenvolvidos na área, mas, ainda, de forma insuficiente para subsidiar decisões públicas.

A relação entre crescimento econômico, desigualdade de renda e pobreza encontra-se no centro do debate de desenvolvimento econômico. Paralelamente a esse debate seguem os 
estudos sobre a relação entre o produto e os investimentos em infraestrutura, como pode-se verificar na sequencia deste trabalho. Indiretamente, tais estudos sugerem alguma relação entre os investimentos em infraestrutura e pobreza. Cabe ressaltar que a pobreza pode se constituir num fator determinante para o colapso de Estados, bem como dos conflitos internos e externos, conforme ensaiado por CAMPOS (p. 29, 2006). Entretanto, existe uma lacuna na literatura para avaliar a relação entre investimento em infraestrutura e possibilidade de incidência de conflitos.

Desta forma, o presente trabalho busca propor uma metodologia específica quantitativa para avaliar a relação entre investimento em infraestrutura e possibilidade de incidência de conflitos. Busca-se proporcionar uma visão dessa provável relação a partir de um procedimento metodológico quantitativo especifico. Para tanto, procura-se definir uma variável agregada que possa representar um estimador eficiente e consistente da pobreza e combinar dados, a fim de obter uma relação de longo prazo com a ocorrência de conflitos. Por exemplo, metodologicamente, a relação entre Produto Interno Bruto (PIB), a incidência de conflitos e investimentos em infraestrutura em setores-chave da economia, tais como energia elétrica, telecomunicações e transportes, em uma região pré-definida, por meio de estimativas com dados de painel. Caso exista essa relação, em que grau essa infraestrutura explica a minimização da probabilidade do conflito?

Este artigo apresenta, primeiramente, uma sucinta revisão da literatura, teórica e empírica, que já abordou o círculo vicioso pobreza e conflitos e a infraestrutura sob a ótica do desenvolvimento regional. Na sequência, apresentaremos um modelo descrito formalmente, que possa descrever a relação de um conjunto de variáveis explicativas com algumas características. Propõe-se investigar, ainda, a causalidade de Granger, verificando se mudanças na variável infraestrutura efetivamente precedem temporalmente mudanças na variável produto. Assim, obtém-se a nossa equação de teste, que poderá ser utilizada para as estimações em trabalhos futuros. Posteriormente, descreveremos a metodologia sugerida na adoção do modelo considerado neste artigo e, na sequência, os resultados decorrentes do modelo e da metodologia. Finalizando com algumas considerações finais e propostas de extensão do presente trabalho.

\section{2 - Revisão da Literatura}

BRADY (2003) analisa problemas das medições da pobreza feitas nos EUA e examina vários avanços teóricos e metodológicos na medição da pobreza, sugerindo a medição ideal da pobreza. De forma mais incisiva sobre a nossa temática de conflitos e pobreza CAMPOS (pp. 
32-33, 2006) menciona que $80 \%$ dos 20 países mais pobres incorreram em conflitos e traça uma relação inversa entre PIB e a probabilidade de ocorrência de conflitos.

Na esteira da visão de ADDISON (2003), pela metodologia proposta, o estudo se desenvolve com a finalidade de apontar um instrumento valioso na tomada de decisões e nas políticas sociais orientadas a minimizar a pobreza, objetivando não apenas reconstrução econômica, mas também fortalecer acordos sócio, político e econômicos. Dessa forma, os investimentos em infraestrutura seriam o início do restabelecimento das instituições e a consequente manutenção da paz.

AGOSTINI, BROWN e GÓNGORA (2008), se utilizam de uma metodologia que considera a distribuição espacial da pobreza no Chile, visando produzir estimadores consistentes da pobreza, relacionando-a ao conjunto de características locais.

O debate sobre crescimento econômico versus desigualdade de renda e pobreza foi enfatizado de 1950 a 1970 (SHORROCKS e HOVEN, 2005), mas não significa que deixou de ser polêmico.

Desde de ASCHAUER (1989) e MUNNELL (1990) um número considerável de trabalhos tem sido publicado dando ênfase ao papel do capital público no desenvolvimento econômico, acreditando que a redução da produtividade seria induzida pela baixa expansão dos investimentos públicos em infraestrutura. $\mathrm{O}$ artigo do primeiro autor foi o precursor na abordagem dessa questão, a partir de dados da economia americana, ele estimou, por mínimos quadrados ordinários (MQO), que um aumento de 1\% do capital público acarretaria um aumento entre $0,36 \%$ e $0,39 \%$ no produto. Munnel, com dados regionais da economia americana, chega a resultados semelhantes. Além disso, UCHIMURA e GAO (1993) chegaram a uma elasticidade do PIB com relação à dotação de capital de infraestrutura de 0,19 para a Coréia e 0,24 para Taiwan.

Estudando o impacto do capital de infraestrutura em países em desenvolvimento, EASTERLY e REBELO (1993) estimaram a elasticidade-renda do investimento em transporte e comunicações entre 0,59 e 0,66 . Os autores realizaram essa análise considerando as variáveis desagregadas, a partir de dados cross-section.

INGRAM (1994), utilizando medidas físicas de estoque de capital de infraestrutura, confirmou a existência de relação entre o capital de infraestrutura e o produto no longo prazo. Seu estudo foi realizado com dados de 100 (cem) países em desenvolvimento.

BENITEZ (1997) demonstra a participação e a colaboração das infraestruturas no desenvolvimento regional, a partir da relação entre o produto e a produtividade regional e as diferentes dotações de infraestruturas existentes, apresentando resultados similares a outros 
trabalhos, inclusive em termos de magnitude da elasticidade obtida. O autor constrói indicadores das dotações regionais com base nas metodologias de BIEHL (1986) e da análise fatorial.

FERREIRA e MALLIAGROS (1998) confirmam para o caso brasileiro a existência de uma forte relação entre infraestrutura e produto de longo prazo. Eles estimaram uma elasticidade-renda de longo prazo entre 0,55 e 0,61 ; com os setores de energia elétrica e transportes influenciando mais intensamente o PIB. Estimam, também, altas elasticidades da produtividade total dos fatores em relação ao capital em infraestrutura. O método da estimação empregado pelos autores foi a análise de cointegração por JOHANSEN (1991, 1995).

KIM (1998) após analisar os efeitos das políticas de investimentos públicos em infraestrutura de transporte sobre o produto, na economia coreana, concluiu que existem vantagens e desvantagens, dependendo das restrições impostas à entrada de capital externo e das alternativas de financiamento dos projetos de infraestrutura. $\mathrm{O}$ autor constatou que gerar inflação se constitui em uma possível desvantagem do investimento em infraestrutura de transporte, só não ocorrendo tal efeito quando os gastos fossem completamente financiados pela receita tributária.

\section{1 - Base de dados}

A base de dados utilizada no presente trabalho foi construída a partir da base de dados do Banco Mundial, que consiste em 15 (quinze) países5, que se localizam em regiões com algum registro de conflitos ou com características concorrentes para a incidência desse evento conflito. O período considerado foi entre os anos 2001 e 2016, inclusive.

O recorte regional das unidades individuais (os países) deve-se ao fato de as informações disponibilizadas na base de dados do Banco Mundial são bem caracterizadas por especificidades de cada país. Isso possibilita a construção de um vetor característico do país, como representado pela variável $H_{i t}$ (conjunto de características local).

O período supracitado foi selecionado de acordo com o contexto sócio histórico de grande incidência do evento de interesse da análise e sem indicativos de rupturas dentro do

\footnotetext{
${ }^{5}$ São países em situação de fragilidade e/ou afetados por conflitos: Afeganistão, Eritreia, Egito, Iraque, Líbia, Nigéria, República Árabe da Síria, Ucrânia, Israel, Quênia, Federação da Rússia, Somália, Tunísia, Serra Leoa e Haiti.
} 
período, que sugere ausência de quebra estrutural. Ainda que a análise proposta possa apresentar limitações, tendo em vista as diferenças de estrutura e de contexto de cada país, existe um avanço na análise consubstanciado na dimensão temporal e regional da investigação, com foco em cada país.

No próximo item será apresentado a descrição das variáveis consideradas no modelo econométrico do indicador.

\section{3 - Aspectos teóricos e metodológico}

\section{1 - Modelo básico}

A figura que se segue realiza um esboço, de forma preliminar, de possíveis relações entre as variáveis que comporão o modelo utilizado. Essas relações preditas intuitivamente não pretendem esgotar causalidades esperadas existentes entre o efetivo investimento em infraestrutura pública e a deflagração de conflitos nos países contemplados no presente estudo. Assim, teremos apenas o desenho provável dos resultados a serem estimados, sem acarretar fragilidade mútua entre esse esboço e as estimações.

Figura 1: Diagrama causal sobre a relação entre infraestrutura e a incidência de conflitos




Fonte: Elaboração própria.

A primeira etapa da metodologia consiste na especificação do modelo básico expresso na equação (1), que caracterize as variáveis utilizadas no modelo econométrico, cuja descrição das variáveis encontra-se no quadro seguinte.

Quadro 1 - Variáveis utilizadas no modelo econométrico (2001-2016)

\begin{tabular}{|c|c|c|}
\hline Variável & Descrição & $\begin{array}{c}\text { Sinal } \\
\text { esperado }\end{array}$ \\
\hline$R$ & $\begin{array}{l}\text { Representa Investimento em infraestrutura }- \text { energia, } \\
\text { telecomunicação, transporte e água. }\end{array}$ & - \\
\hline$D$ & $\begin{array}{l}\text { Expressa as características demográficas do lugar } \\
\text { (densidade demográfica, taxa de crescimento populacional, } \\
\text { percentual de população urbana, participação do gênero } \\
\text { masculino e feminino na população, separadamente, por } \\
\text { faixa etária) }\end{array}$ & + \\
\hline$H$ & $\begin{array}{l}\text { Representa o vetor característico da região/país (conjunto de } \\
\text { características local), tais como percentual da população na } \\
\text { linha da pobreza, taxa de natalidade, taxa de mortalidade de } \\
\text { adultos, número de refugiados por país de origem, índice de } \\
\text { desigualdade da distribuição de renda e despesas per capita } \\
\text { (públicas e privadas) com saúde. }\end{array}$ & - \\
\hline Conf & $\begin{array}{l}\text { Representa a proxy da variável dependente, representada } \\
\text { pelo número de pessoas mortas relacionadas a conflitos no } \\
\text { país } i \text { no ano } t \text {. }\end{array}$ & + \\
\hline$D T$ & $\begin{array}{l}\text { Variáveis dummies de tempo (com periodicidade de } 1 \text { em } 1 \\
\text { ano, até o último ano de interesse). }\end{array}$ & + \\
\hline DRe & Dummy regional (do país) & + \\
\hline
\end{tabular}

Fonte: elaboração própria. Nota: Os valores monetários foram deflacionados com base no ano 2000.

$$
\operatorname{Conf}_{i t}=\beta_{0}+\beta_{1} D_{i t}+\beta_{2} H_{2 i t}+\beta_{3} R_{3 i t}+u_{i t},
$$

onde Conf $_{i t}$ representa a proxy da variável dependente, representada pelo número de pessoas mortas relacionadas a conflitos no país $i$ no ano $t$. A variável $D_{i t}$ expressa as características demográficas do lugar (densidade demográfica, taxa de crescimento populacional, percentual 
de população urbana, participação do gênero masculino e feminino na população, separadamente, por faixa etária); $H_{2 i t}$ este representa o vetor característico da região/país (conjunto de características local), tais como percentual da população na linha da pobreza, taxa de natalidade, taxa de mortalidade de adultos, índice de desigualdade da distribuição de renda e despesas per capita (públicas e privadas) com saúde. $R_{3 i t}$ a infraestrutura. Por último, temos o termo de erro $u_{i t}$. As variáveis explicativas podem ser acrescidas de outras que possam atribuir consistência àquela que se busca explicar Confit, por exemplo, carga tributária, IDH, taxa líquida de imigração, dentre outras.

Conforme verificado pela revisão de literatura, a taxa de crescimento do produto interno bruto (PIB) per capita poderia expressar não apenas o crescimento econômico, mas o desenvolvimento, devido a sua conotação de bem-estar, pois a precariedade dos índices relativos às variáveis são fatores determinantes da pobreza, com incremento na probabilidade de ocorrência de conflitos. Dessa forma, se utilizássemos o PIB como variável explicativa da proxy de conflito, como são conjuntamente dependentes, elas estariam correlacionadas com os termos de erro. Além disso, a literatura citada no início do trabalho sugere correlação da variável infraestrutura com a variável PIB, ambas como preditoras. Essas questões tornam os métodos clássicos de mínimos quadrados ordinários (MQO) inaplicáveis à estimativa dos parâmetros da equação.

\section{2 - Modelo de regressão com dados em painel}

A metodologia básica consiste em realizar regressões por meio dos Mínimos Quadrados Ordinários a partir de dados em painel (também chamados de dados combinados) (combinação de séries temporais e observações em corte transversal). Na equação (1) o $i$ representa a $i$-ésima unidade de corte transversal (país) e o $t$ o $t$-ésimo período de tempo.

Vimos que os modelos de regressão com dados em painel agregam uma combinação de séries temporais e de observações em corte transversal multiplicadas por $\mathrm{T}$ períodos de tempo. Esses modelos proporcionam mais informação e viabilizam a realização de um estudo da dinâmica da mudança do fenômeno investigado. Além disso, teremos mais variabilidade e menos colinearidade entre as variáveis, bem como mais graus de liberdade.

A partir da descrição acima, os dados em painel são mais informativos, apresentam mais variabilidade e menos colinearidade. Mas, podem ocorrer problemas relacionados à seleção de dados, que não se configuram em uma amostra aleatória. Para a correção necessária do viés resultante dessa seleção da amostra não aleatória os modelos em painel 
podem utilizar as abordagens dos efeitos fixos e dos efeitos aleatórios. Além disso, no presente trabalho, realizaremos os testes de robustezes para a escolha do melhor modelo econométrico.

No presente artigo, a metodologia básica consiste em realizar, primeiramente, regressões em painel dos efeitos fixos decorrem do fato de que os respectivos interceptos individuais não se alteram ao longo do tempo (invariante no tempo), ainda que os interceptos possam ser diferentes entre os indivíduos. Na equação 2 , que se segue, o $\beta_{1 i}$ sugere que o intercepto de cada país (ou indivíduo) é invariante no tempo. Assim, especificação por variáveis de tempo pode ser controlada na forma:

$$
\operatorname{conf}_{i t}=\alpha_{0}+\gamma_{1}+\gamma_{2}+\ldots+\gamma_{t-1}+\beta_{1 i}+\beta_{1} x_{1 i t}+\beta_{2} x_{2 i t}+\ldots+\beta_{n} x_{n i t}+u_{i t}
$$

onde conf é o vetor das taxas de crescimento da mortalidade por conflito de cada país em cada período (o subscrito $i$ se refere ao país, que representa a unidade de corte transversal, e $t$ período de tempo em ao ano), $\gamma_{1}, \gamma_{1}, \ldots, \gamma_{t}$ são os vetores das variáveis dummies de tempo (com periodicidade de 1 em 1 ano, até o último ano de interesse) e $x_{1 i t}, x_{2 i t}, \ldots, x_{n i t}$ são os vetores das variáveis explicativas não estocásticas e o termo de erro segue a premissa clássica de $E\left(u_{i t}\right) \sim N\left(0, \partial^{2}\right)$.

O modelo do efeito fixo da equação 2 pressupõe que os coeficientes angulares dos regressores (variáveis do lado direito) da equação não variam entre os indivíduos (países considerados no trabalho).

A modelagem de feitos fixos geralmente é exigente em termos de graus de liberdade, se houver muitas unidades de corte transversal (países). Um exemplo dessa perda de graus de liberdade observa-se no modelo de variáveis binárias (ver detalhes em KMENTA (1986).

Na medida em que o modelo de variáveis binárias expressa uma falta de conhecimento sobre o modelo verdadeiro, podemos especificar um modelo que expresse essa falta de conhecimento por meio do termo de erro $u_{i t}$. Dessa forma, testaremos a abordagem dos modelos de correção de erros ou modelos de efeitos aleatórios.

Para levarmos em consideração o modelo de efeitos aleatórios começaremos pela equação (2), sem as dummies:

$$
\operatorname{conf}_{i t}=\beta_{1 i}+\beta_{1} x_{1 i t}+\beta_{2} x_{2 i t}+\ldots+\beta_{n} x_{n i t}+u_{i t} \beta_{1 i}+\beta_{1} x_{1 i t}+\beta_{2} x_{2 i t}+\ldots+\beta_{n} x_{n i t}+u_{i t}
$$


Não trataremos o $\beta_{l i}$ como fixo, mas sim como uma variável aleatória com valor médio (sem lançar o subscrito $i$ ). Assim, o valor do intercepto para um país é representado por:

$$
\beta_{1 i}=\beta_{1}+\varepsilon_{i} \quad i=1,2, \ldots, N
$$

onde $\varepsilon_{i}$ é um termo de erro aleatório com média zero e variância $\partial^{2}$.

Com a combinação descrita na equação (3) estamos afirmando que retiramos a nossa amostra de países de um universo maior do que a utilizada nas análises, e que os países têm um valor médio comum para o intercepto (igual a $\beta_{1}$ ). Além disso, estamos afirmando que o as diferenças individuais no intercepto de cada país é expresso no termo do erro $\varepsilon_{i}$.

Substituindo (4) em (3), obtemos:

$$
\begin{aligned}
& \operatorname{conf}_{i t}=\beta_{1}+\beta_{1} x_{1 i t}+\beta_{2} x_{2 i t}+\ldots+\beta_{n} x_{n i t}+u_{i t} \beta_{1 i}+\beta_{1} x_{1 i t}+\beta_{2} x_{2 i t}+\ldots+\beta_{n} x_{n i t}+\varepsilon_{i}+u_{i t} \\
& =\beta_{1}+\beta_{1} x_{1 i t}+\beta_{2} x_{2 i t}+\ldots+\beta_{n} x_{n i t}+u_{i t} \beta_{1 i}+\beta_{1} x_{1 i t}+\beta_{2} x_{2 i t}+\ldots+\beta_{n} x_{n i t}+w_{i t}
\end{aligned}
$$

onde

$$
w_{i t}=\varepsilon_{i}+u_{i t}
$$

Dessa forma, incluímos no termo de erro $w_{i t}$ é composto por elemento do corte transversal, $\varepsilon_{i}$ (de cada indivíduo ou país) e elemento combinado da série temporal e do corte transversal $\left(u_{i t}\right)$. Ressaltando-se que não podemos olvidar os pressupostos clássicos normalidade relacionados à média e variância no modelo acima obtido de correção de erros.

Para que não haja a mera suposição na escolha do melhor modelo, a decisão entre o uso do modelo de efeitos fixos e do modelo de correção de erros é realizada por meio do teste de Hausman (ver detalhes em HAUSMAN (1978)).

É importante salientar que o uso de dados em painel em estudos de crescimento econômico permite fazer um controle para a omissão de variáveis que estão presentes ao longo do tempo. 
No presente trabalho, a análise dos fatores que sugerem a determinação de incidência de conflitos nos países será realizada com base no modelo econométrico a ser estimado, na forma empilhada, representado pela combinação entre as equações 1, 2 e 5.

\section{4 - Resultados e discussões}

Para os resultados do modelo em dados em painel, a partir do modelo econométrico (equação 5), foram estimados sequencialmente, o modelo de dados agrupados por MQO $(O L S)$, o modelo de efeitos aleatórios e o modelo de efeitos fixos. Os resultados das estimações e de todos os testes realizados encontram-se na Tabela 1. Logo após a estimação por MQO, realizou-se o teste de heterocedasticidade (Breusch-Pagan/Cook-Weisberg). O resultado, por sua vez, não rejeitou a hipótese nula de homocedasticidade.

Em relação a presença/ausência de efeitos não observados, observa-se que o teste de Breusch-Pagan (BP), realizado posteriormente às estimações do modelo com efeitos aleatórios, foi significativo a 1\%, o qual rejeita-se a hipótese nula de ausência de efeitos não observados. Nesse caso, deve-se considerar que os países brasileiros possuem muitas características peculiares à sua formação e, muitas dessas caracterizações não são observadas e podem variar entre as unidades cross-section (heterogeneidade não observada). Caso esses fatores não observados influenciem e sejam correlacionadas com as variáveis de interesse, os efeitos estimados destas variáveis serão tendenciosos.

Ressalta-se que os efeitos não observados podem ser modelados por meio dos efeitos fixos ou aleatórios. O teste de Hausman, no qual se rejeitou a hipótese nula de que os efeitos aleatórios são consistentes, apontou que a melhor seleção é a modelagem por efeitos fixos. Da mesma forma, deve-se levar em consideração que o método de estimação por MQO pode apresentar o viés de heterogeneidade e o de variável omitida (CAMERON e TRIVEDI, 2005).

Tabela 1 - Resultados das estimações realizadas para o modelo em dados em painel

\begin{tabular}{|c|c|c|c}
\hline \multirow{2}{*}{ C\&T } & $\begin{array}{c}\text { Dados } \\
\text { Empilhados } \\
\text { (MQO) }\end{array}$ & $\begin{array}{c}\text { Efeito } \\
\text { Aleatório }\end{array}$ & Efeito Fixo \\
\cline { 2 - 4 } & \multicolumn{3}{|c}{ Coeficientes } \\
\hline PIB per (mil) & $-1.9600^{* *}$ & $-16.3175^{* *}$ & $-19.2141^{* *}$ \\
$(-3.73)$ & $(-4.33)$ & $(-4.10)$
\end{tabular}


RELACult - Revista Latino-Americana de Estudos em Cultura e Sociedade

Revista Latinoamericana de Estudios en Cultura y Sociedad | Latin American Journal of Studies in Culture and Society V. 03, ed. especial, dez., 2017, artigo n ${ }^{\circ} 627$ | relacult.claec.org | e-ISSN: 2525-7870

\begin{tabular}{|c|c|c|c|}
\hline$R$ Energia (mil) & $\begin{array}{c}-1.6730 * * \\
(-2.14)\end{array}$ & $\begin{array}{c}-3.3134 * * \\
(-2.94)\end{array}$ & $\begin{array}{c}-5.3248 * * \\
(-4.59)\end{array}$ \\
\hline$R$ Telecom & $\begin{array}{c}-8.7833 * * \\
(-3.95)\end{array}$ & $\begin{array}{c}-3.4044 \\
(-1.56)\end{array}$ & $\begin{array}{c}-3.7358^{*} \\
(-1.72)\end{array}$ \\
\hline Conf & $\begin{array}{c}0.7464 * * \\
(13.33)\end{array}$ & $\begin{array}{l}0.7441 \\
(1.29)\end{array}$ & $\begin{array}{l}0.8350 \\
(1.07)\end{array}$ \\
\hline$R$ transport (mil) & $\begin{array}{c}-0.5301 * \\
(-1.73)\end{array}$ & $\begin{array}{l}-0.1734 \\
(-0.70)\end{array}$ & $\begin{array}{c}-0.1668 \\
(-0.73)\end{array}$ \\
\hline$D$ & $\begin{array}{c}0,7145 \\
(1,7524)\end{array}$ & $\begin{array}{c}0,8178 \\
(2,0456)\end{array}$ & $\begin{array}{c}0,9513 \\
(2,1036)\end{array}$ \\
\hline$H$ & $\begin{array}{c}0,4143 \\
(1,0367)\end{array}$ & $\begin{array}{c}0,5246 \\
(2,0135)\end{array}$ & $\begin{array}{l}0,71312 \\
(2,8213)\end{array}$ \\
\hline Rágua (mil) & $\begin{array}{c}-0.4952 \\
(-1.36)\end{array}$ & $\begin{array}{c}-0.4686 * * \\
(-3.39)\end{array}$ & $\begin{array}{c}-0.4649 * * \\
(-3.80)\end{array}$ \\
\hline$\beta 0$ & $\begin{array}{c}8,0145 \\
(1,8512)\end{array}$ & $\begin{array}{c}7,8276 \\
(2,4561)\end{array}$ & $\begin{array}{c}6,0514 \\
(3,1025)\end{array}$ \\
\hline$\beta 1$ & $\begin{array}{c}0,5145 \\
(1,0356)\end{array}$ & $\begin{array}{l}0,61235 \\
(2,0158)\end{array}$ & $\begin{array}{l}0,81301 \\
(2,7204)\end{array}$ \\
\hline$\beta 2$ & $\begin{array}{c}-0,6245 \\
(-1,5246)\end{array}$ & $\begin{array}{c}-0,8245 \\
(-2,0513)\end{array}$ & $\begin{array}{c}-0,7845 \\
(-3,1542)\end{array}$ \\
\hline$\beta 3$ & $\begin{array}{c}0,3532 \\
(1,3251)\end{array}$ & $\begin{array}{l}0,3934 \\
1,4512)\end{array}$ & $\begin{array}{l}0,4427 \\
(2,381)\end{array}$ \\
\hline cons & $\begin{array}{c}-2.2780 * * \\
(-3.11)\end{array}$ & $\begin{array}{c}-2.0845 * * \\
(-2.61)\end{array}$ & $\begin{array}{c}-2.0338 * * \\
(-2.89)\end{array}$ \\
\hline $\mathrm{R}^{2}$ & 0.7041 & 0.2703 & 0.3201 \\
\hline Breusch Pagan & \multicolumn{3}{|c|}{$177.84 * *$} \\
\hline
\end{tabular}




\begin{tabular}{|c|c|}
\hline $\begin{array}{l}\text { Teste de } \\
\text { Heterocedasticidade } \\
\text { (Breusch-Pagan/ } \\
\text { Cook-Weisberg) } \\
\operatorname{chi}^{2}(1)\end{array}$ & 1.22 \\
\hline Teste Chow & $7.93 * *$ \\
\hline
\end{tabular}

Nota: $* \mathrm{e}^{* *}$, são significativos a $5 \%$ e $10 \%$, respectivamente. Os coeficientes sem asterisco são significativos a $1 \%$.

A tabela acima apresenta os valores estimados das variáveis correspondentes ao investimento em infraestrutura (energia, telecomunicação, transporte e água), $R$ telecom, $R$ transport, $R$ água e $R$ energia, apresentam sinal negativo (esperado), conforme descrito no Quadro 1 (descrição de variáveis). Enquanto o coeficiente da variável conflito (Conf) sugere uma relação inversa com as variáveis correspondentes ao investimento em infraestrutura. Nesse contexto, se considerarmos o modelo em painel de efeitos fixos, existe uma correlação negativa entre Conf e investimento em infraestrutura.

$\mathrm{O}$ valor estimado de 0.8350 referente à variável Conf sugere uma probabilidade elevada de incidência de conflitos. Podemos somar os valores correspondentes a cada variável de infraestrutura, o resultado será de 9,6923. Dessa soma depreendemos que a cada 9,6 mil dólares teremos uma probabilidade de 0,8350 de ocorrência de conflito.

Além disso, por analogia, teremos uma correlação negativa entre o crescimento do PIB e a incidência de conflito. Os resultados sugerem que a redução no nível do PIB aumenta a possibilidade de ocorrência de conflito. Existe uma consistência entre os valores negativos estimados do investimento em infraestrutura e o sinal de negativo do valor estimado no PIB, consubstancia e confirma a correlação positiva entre investimento em infraestrutura e PIB. Essa consistência corroborada os resultados da literatura sobre a elasticidade-renda desse investimento em relação ao PIB.

Ademais, os correspondentes aos índices de desigualdade de renda, concentração de renda e outros componentes sociais representados na variável $H$ apresentam sinal negativo. Desse resultado depreende-se que em situação desfavorável apresentada pelos elementos constituintes dessa variável potencializa-se a incidência de conflitos nos países considerados.

\section{4 - Considerações finais}

O trabalho teve como objetivo mostrar a ligação entre pobreza e a incidência de conflitos como um ciclo vicioso no qual a pobreza gera conflitos e conflitos geram pobreza. 
Dessa forma, foi imprescindível pensar que, no esforço de análise e prevenção de conflitos, devem ser levadas em ampla consideração as ameaças provenientes dos aspectos estruturais sociais e econômicos historicamente reproduzidos nos países menos desenvolvidos. Nesse contexto, propusemos uma metodologia específica para avaliar a relação entre investimento em infraestrutura e possibilidade de incidência de conflitos.

Para tanto, analisamos, empiricamente, a relação entre a incidência de conflitos e investimentos em infraestrutura em setores-chave da economia, tais quais energia elétrica, telecomunicações e transportes, em uma região pré-definida, por meio de estimativas com dados de painel. Essa relação foi mensurada principalmente por meio da estimação de parâmetros que expressam o impacta (negativo ou positivo) da infraestrutura (logística pública e privada) sobre a incidência de conflitos. Assim, obteve-se evidências empíricas indicando que investimentos, no que se conhece como logística pública e privada, podem contribuir para o crescimento econômico da região/país, redução da pobreza e, por consequência, reduzir a incidência de conflitos.

Mais especificamente, os resultados econométricos dessa análise sugerem consistência com a literatura que analisa a elasticidade-renda do investimento em infraestrutura. Além disso, apesar da diminuta literatura sobre a relação entre investimento em infraestrutura e a probabilidade de incidência de conflitos, os resultados sugerem um significante efeito conjunto de investimentos na referida infraestrutura em relação ao PIB e sobre a ocorrência de conflitos.

Diante do exposto, conclui-se a metodologia - e, dessa forma, o objetivo desse artigo - com a demonstração de que as condições estruturais podem representar as raízes dos conflitos em que se envolvem os países que se escolha analisar.

Finalmente, propõe-se, como extensão do presente trabalho, investigar, ainda, a causalidade de Granger, verificando se mudanças na variável infraestrutura efetivamente precedem temporalmente mudanças na variável Conf (conflito). Assim, nessa extensão, propõe-se testar a causalidade de Granger para a relação entre o crescimento do PIB e a probabilidade de ocorrência de conflitos.

\section{Referências}

ADDISON, T. (2003). Africa's Recovery from Conflict: Making Peace Work for the Poor. UNU-Wider. Disponível em: <http://www.wider.unu.edu/publications/policybriefs/en_GB/pb6/_files/78807311704195264/default/pb6.pdf>. Acesso em: 04 set. 2017. 
AGOSTINI, C., P. H, BROWN e D. P., GÓNGORA (2008). Nota Técnica: Desigualdad Geográfica en Chile, Revista de Análisis Económico, v. 35, n. 1, p. 79-110, jun 2008.

ASCHAUER, D. A. (1989). Public investment and productivity growth in the Group of Seven. Economic Perspectives 13, p.17-25.

BALTAGI, B. H. Econometrics analysis of panel data. 2 ed. Chichester, UK: Wiley \& Sons, 2001.

BALTAGI, B. H. (2002). Econometric Analysis of Panel Data. John Wiley \& Sons: England.

Banco Mundial (1994). World Development Report - Infrastructure for Development Report, n. 134831. Disponível em: <http:// www.worldbank.org./ .> Acesso em: 04 set. 2017.

Banco Mundial (2002). World Development Report - Brazil: The New Growth Agenda, n. 22950-BR, Disponível em: <http:// www.worldbank.org./ .> Acesso em: 04 set. 2017.

BENITEZ, R. M. (1997). A participação das infra-estruturas nas disparidades regionais - um estudo empírico. Curitiba, Dissertação de mestrado- UFPR.

BRADY, D. (2003), Rethinking the sociological measurement of poverty. Workin Paper. Durham, Duke University. BRANTE, Thomas. (2001) Consequências do realismo na construção de teoria sociológica. Oeiras, Sociologia, set., no.36, p.9-38

BRAUN, J. (2007). Rural-Urban Linkages for Growth, Employment, and Poverty Reduction, International Food Policy Research Institute. Ethiopian Economic Association, Fifth International Conference on the Ethiopian Economy, June.

BRIGAGÃO, C. e CAMPOS DE MELLO, V. (org.) (2006). Diplomacia cidadã: panorama brasileiro de prevenção de conflitos internacionais. Rio de Janeiro: Gramma: Fundação Konrad Adenauer.

CAMERON, A.C., TRIVEDI, P.K. Microeconomics: Methods and Applications. Cambridge : Cambridge University Press, 2005.

DÉMURGER, S. (2001). Infrastructure development and economic growth: an explanation for regional disparities in China? Journal of Comparative Economics 29, 95-117.

EASTERLY, W e S. REBELO (1993). Fiscal policy and economic growth: an empirical investigation. Journal of Monetary Economics, v. 32, p. 417-458.

ESFAHANI, H. S. e M. T. RAMIREZ (2003). Institutions, infrastructure, and economic growth. Journal of Development Economics 70, 443-477.

FERREIRA, P. C. (1996) Investimento em Infra-estrutura no Brasil: Fatos Estilizados e Relações de Longo Prazo. Pesquisa e Planejamento Econômico, v.26, n.2, p.231- 252, Ago.1996.

FERREIRA, P. C. e T. G. MALLIAGROS (1998). Impactos produtivos da Infra-estrutura no Brasil: 1950-1995. Pesquisa e Planejamento Econômico, vol. 2, pp. 315-338, Fundação Getúlio Vargas, 1998.

FIGUEIREDO, A. (2006). Como Preparar um Trabalho para Apresentação em Congressos de Pesquisa e Publicações Científicas. Disponível em: < www.ucb.br/pesquisa. > Acesso em: 05 jun 2017.

GREENE, W. H. (1997). Econometric Analysis. Prentice-Hall: United States of America.

HAUSMAN, J. A., Specification tests in Econometrics. Econometrica, vol. 46, 1978, p. 12511271. 
HERRANZ-LONCAN, A. (2007). Infrastructure investment and Spanish economic growth, 1850-1935. Explorations in Economic History 44(3), 452-468.

HOLTZ-EAKIN, D. e A. E. SCHWARTZ (1995). Infrastructure in a Structural Model of Economic Growth. Regional Science and Urban Economics 25, 131-151.

IM, K. S., M. H. PESARAN e Y. SHIN (2003). Testing for unit roots in heterogeneous panel. Journal of Econométrica, 115 (2003) 53-74.

INGRAM, G. (1994). Sustaining Infrastructure to Support Economic Growth. World. Bank, minmeo.

KIM, E. (1998). Economic Gain and Loss from Public Infrastructure Investment, Growth and Change 29 (4), 445-469.

KMENTA, JAN. Elemnts of Econometrics. 2. ed. Nova York: Macmillan, 1986.

LAU, S-H. P. e C-Y. SIN (1997). Public Infrastructure and Economic Growth: Time-Series Properties and Evidence. The Economic Record 73, 125-135.

LEVIN, A. e C. F. LIN (2002). Unit Root Test in Panel Data: Asymptotic and Finite Sample Properties. Journal of Econométrica, 108 (2002) 1-24.

MUNNELL, A. H. (1990). How Does Public Infrastructure Aect Regional Economic Performance? New England Economic Review, september, p; 11-32.

PÊGO FILHO, B., J. O. CÂNDIDO JÚNIOR e F. PEREIRA (1999). Investimento $e$ Financiamento da Infra-Estrutura no Brasil: 1990-2002. Texto para Discussão n. 680, Instituto de Pesquisa Econômica Aplicada - IPEA, Brasil.

ROGOFF, K. (2004) É a Chance do Brasil. Veja, São Paulo, ed. 1862, 14 jul 2004. p. 11, 14 e 15. Entrevista.

SCHEINKMAN, J. A., M. B. Lisboa, et al. (2002). A Agenda Perdida: diagnósticos e propostas para retomada do crescimento com maior justiça social. < Disponível em: http://www.econ.puc-rio.br > Acesso em: 04 set. 2017.

UCHIMURA, K. e H. GAO (1993). The Importance of Infrastructure on Economic Development, Mimeo, World Bank.

WANG, E. C. (2002). Public infrastructure and economic growth: a new approach applied to East Asian economies. Journal of Policy Modeling (24), 411-435

WOOLDRIDGE, J. Econometric analysis of cross section and panel data. Cambridge, Mass: MIT Press, 2002.

WYLIE, P. J. (1996). Infrastructure and Canadian Economic Growth 1946-1991 The Canadian Journal of Economics 29, 350-355. 